\title{
A NEW PROOF OF SONINE'S FORMULA
}

\author{
KRZYSZTOF STEMPAK
}

(Communicated by George R. Sell)

\begin{abstract}
We give a new proof of an old integral formula due to Sonine in which the Bessel functions are involved. By considering the Banach algebra of radial functions on $\mathbf{R}^{n}, n \geq 2$, we observe that Sonine's formula is valid for all positive integers. Next, a complex function theory argument is applied to obtain the validity of the formula for all complex parameters $z$ with $\operatorname{Re} z>0$.
\end{abstract}

Among the huge quantity of formulae listed in Watson's classical monograph [4] there is the following one (cf. [4, p. 411]) due to Sonine:

(1) $\int_{0}^{\infty} J_{\nu}(a t) J_{\nu}(b t) J_{\nu}(c t) t^{1-\nu} d t=2^{\nu-1} \Gamma\left(\nu+\frac{1}{2}\right)^{-1} \Gamma\left(\frac{1}{2}\right)^{-1} \frac{\Delta(a, b, c)^{2 \nu-1}}{(a b c)^{\nu}}$.

$J_{\nu}$ denotes here the Bessel function of order $\nu, \operatorname{Re} \nu>-\frac{1}{2}$, and $\Delta(a, b, c)$ means the area of a triangle with sides $a, b, c>0$. If such a triangle does not exist then the integral in (1) equals 0.

The aim of this note is to prove (1) using a group-theoretic argument and a result which is an application of a theorem of Phragmén-Lindelöf type. The idea of using a theorem of such type has been suggested to the author in a conversation with Andrzej Hulanicki to whom I am very indebted.

As a matter of fact, we will apply the following

THEOREM [3, p. 186]. Suppose $f(z)$ is an analytic function on $\operatorname{Re} z>-\delta$, $\delta>0$, which is of the form $O\left(e^{k|z|}\right)$ on $\operatorname{Re} z \geq 0$, where $k<\pi$. If $f(z)=0$ for $z=0,1,2, \ldots$ then $f=0$ identically.

Now, for $x, y \geq 0$ and $\operatorname{Re} z>0$ denote

$$
\phi^{(z)}(t)=(2 / t)^{(z-1) / 2} \Gamma((z+1) / 2) J_{(z-1) / 2}(t), \quad t>0,
$$

and

$$
\begin{array}{r}
W_{x, y}^{(z)}(t)=2^{z-2} \Gamma\left(\frac{z+1}{2}\right)\left(\Gamma\left(\frac{z}{2}\right) \Gamma\left(\frac{1}{2}\right)\right)^{-1} \frac{\Delta(x, y, t)^{z-2}}{(x y t)^{z-1}} t^{z} \\
|x-y|<t<x+y .
\end{array}
$$

In [2] it has been shown how an application of a version of the Hankel inversion formula to (1) gives

$$
\phi^{(z)}(a x) \phi^{(z)}(a y)=\int_{|x-y|}^{x+y} \phi^{(z)}(a t) W_{x, y}^{(z)}(t) d t,
$$

Received by the editors October 14, 1986.

1980 Mathematics Subject Classification (1985 Revision). Primary 33A40; Secondary 33A75.

Key words and phrases. Bessel functions, radial functions. 
where $a, x, y \geq 0$ and $\operatorname{Re} z>0$. In fact the same argument allows us to deduce (1) from (2). Thus, instead of showing (1) we will prove (2). Note, that by homogeneity it suffices to verify (2) for $a=1$ only.

So, fix $x, y \geq 0$ and consider the functions

$$
F(z)=\phi^{(z)}(x) \phi^{(z)}(y) \text { and } G(z)=\int_{|x-y|}^{x+y} \phi^{(z)}(t) W_{x, y}^{(z)}(t) d t
$$

which are, clearly, analytic on $\operatorname{Re} z>0$. We are going to show that $F=G$ identically. To do this remove, first of all, the common term $\Gamma((z+1) / 2)^{2}$ from both $F$ and $G$. Therefore, instead of $F$ and $G$ we consider

$$
\widetilde{F}(z)=\tilde{\phi}^{(z)}(x) \tilde{\phi}^{(z)}(y) \quad \text { and } \quad \widetilde{G}(z)=\int_{|x-y|}^{x+y} \tilde{\phi}_{x, y}^{(z)}(t) \widetilde{W}^{(z)}(t) d t
$$

where

$$
\tilde{\phi}^{(z)}(t)=\frac{\phi^{(z)}(t)}{\Gamma((z+1) / 2)} \quad \text { and } \quad \widetilde{W}_{x, y}^{(z)}(t)=\frac{W_{x, y}^{(z)}(t)}{\Gamma((z+1) / 2)} .
$$

First, estimate $|\widetilde{F}(z)|$ and $|\widetilde{G}(z)|$. Using the formula

$$
J_{\nu}(t)=\left(\frac{t}{2}\right)^{\nu} \Gamma\left(\nu+\frac{1}{2}\right)^{-1} \Gamma\left(\frac{1}{2}\right)^{-1} \int_{0}^{\pi} e^{i t \cos \theta} \sin ^{2 \nu} \theta d \theta,
$$

which is valid for $\operatorname{Re} \nu>-\frac{1}{2}$, we easily observe that for $\operatorname{Re} z>0$,

$$
\left|\tilde{\phi}^{(z)}(t)\right| \leq\left|\Gamma\left(\frac{z}{2}\right)\right|^{-1} \Gamma\left(\operatorname{Re} \frac{z}{2}\right) \Gamma\left(\operatorname{Re} \frac{z}{2}+\frac{1}{2}\right)^{-1} .
$$

Thus, for $\operatorname{Re} z \geq \frac{1}{2}$

$$
\left|\tilde{\phi}^{(z)}(t)\right| \leq C_{0}|\Gamma(z / 2)|^{-1}, \quad C_{0}=\max _{s \geq 1 / 4} \Gamma(s) \Gamma\left(s+\frac{1}{2}\right)^{-1},
$$

which implies

$$
|\widetilde{F}(z)| \leq C|\Gamma(z / 2)|^{-2}, \quad \operatorname{Re} z \geq 1 / 2 .
$$

On the other hand, since

$$
\int_{|x-y|}^{x+y}\left|\widetilde{W}_{x, y}^{(z)}(t)\right| d t=\left|\Gamma\left(\frac{z}{2}\right)\right|^{-1} \Gamma\left(\operatorname{Re} \frac{z}{2}\right) \Gamma\left(\operatorname{Re} \frac{z+1}{2}\right)^{-1} \int_{|x-y|}^{x+y} W_{x, y}^{(\operatorname{Re} z)}(t) d t
$$

and, cf. e.g. [2], for real $s>0$

$$
\int_{|x-y|}^{x+y} W_{x, y}^{(s)}(t) d t=1
$$

then

$$
\int_{|x-y|}^{x+y}\left|\widetilde{W}_{x, y}^{(z)}(t)\right| d t \leq C_{0}\left|\Gamma\left(\frac{z}{2}\right)\right|^{-1}
$$

for all $\operatorname{Re} z \geq \frac{1}{2}$. Combining (3) and (5) gives

$$
|\widetilde{G}(z)| \leq C\left|\Gamma\left(\frac{z}{2}\right)\right|^{-2}, \quad \operatorname{Re} z \geq \frac{1}{2}
$$


But, cf. e.g. [1, p. 51], for $\operatorname{Re} z \geq \frac{1}{2}$ we have

$$
|\Gamma(z)|^{-1} \leq C \exp (\pi|z| / 2)
$$

and therefore we eventually get

$$
|\widetilde{F}(z)| \leq C \exp (\pi|z| / 2) \quad \text { and } \quad|\widetilde{G}(z)| \leq C \exp (\pi|z| / 2)
$$

for $\operatorname{Re} z \geq \frac{1}{2}$.

Now, in the second step, we will show that $\widetilde{F}(z)=\widetilde{G}(z)$, or, what is the same, $F(z)=G(z)$, for $z=1,2,3, \ldots$ To do this consider $L_{r}^{1}\left(\mathbf{R}^{n}\right)$, the commutative Banach algebra (under convolution) of radial, integrable functions on the Euclidean group $\mathbf{R}^{n}, n \geq 2$. A function $\tilde{f}$ on $\mathbf{R}^{n}$ is radial if $\tilde{f}(\bar{x})=f(\|\bar{x}\|), \bar{x} \in \mathbf{R}^{n}$, for a function $f$ on $(0, \infty)$, called the radial part of $\tilde{f}$. Denote $d \mu_{n}(t)=\sigma(n) t^{n-1} d t$ and $L^{1}\left(\mu_{n}\right)=L^{1}\left(\mathbf{R}_{+}, d \mu_{n}\right)$, where $\sigma(n)=2 \pi^{n / 2} \Gamma(n / 2)^{-1}$ is the volume of the sphere $\Sigma_{n-1}=\left\{\bar{x} \in \mathbf{R}^{n}:\|\bar{x}\|=1\right\}$.

It is easily seen that radial part of the convolution

$$
\tilde{f} * \tilde{g}(\bar{x})=\int_{\mathbf{R}^{n}} \tilde{f}(\bar{y}) g(\bar{x}-\bar{y}) d \bar{y}
$$

of two functions $\tilde{f}, \tilde{g} \in L_{r}^{1}\left(\mathbf{R}^{n}\right)$ with the radial parts $f, g$ respectively, is the function $f * g$ given by

$$
f * g(x)=\int_{0}^{\infty} f(y) T^{y} g(x) d \mu_{n}(y), \quad x>0
$$

where the generalized translation $T^{y}, y \geq 0$, is

$$
T^{y} g(x)=\int_{|x-y|}^{x+y} g(t) W_{x, y}^{(n-1)}(t) d t .
$$

(We abuse the notation slightly by using the same symbol $*$ to denote the convolution in $\mathbf{R}^{n}$ and the operation given by (6).) To verify (6) let us introduce the polar coordinates in $\mathbf{R}^{n}$ letting

$$
y_{j}=y \cos \phi_{j} \prod_{i=1}^{j-1} \sin \phi_{i}, \quad j=1,2, \ldots, n-1, \quad y_{n}=y \prod_{i=1}^{n-1} \sin \phi_{i}
$$

where $y>0$ and $0<\phi_{j}<\pi, j=1,2, \ldots, n-2,0<\phi_{n-1}<2 \pi$. The Jacobian of this transformation is

$$
\mathscr{J}=y^{n-1} \mathscr{J}_{0}\left(\phi_{1}, \ldots, \phi_{n-1}\right), \quad \mathscr{J}_{0}=\prod_{j=1}^{n-2} \sin ^{n-j-1} \phi_{j} .
$$

Now, for $x=(x, 0, \ldots, 0), \bar{y}=\left(y_{1}, \ldots, y_{n}\right)$ we get

$$
\begin{aligned}
& \text { (7) } \tilde{f} * \tilde{g}(\bar{x})= \int_{0}^{\infty} f(y) \int_{0}^{2 \pi} \int_{[0, \pi]^{n-2}} \tilde{g}\left(x-y \cos \phi_{1}, \ldots\right) \\
& \times \mathscr{J}_{0}\left(\phi_{1}, \ldots, \phi_{n}\right) d \phi_{1} \cdots d \phi_{n} y^{n-1} d y \\
&=2 \pi^{(n-1) / 2} \Gamma\left(\frac{n-1}{2}\right)^{-1} \int_{0}^{\infty} f(y) \int_{0}^{\pi} g\left(\left(x^{2}+y^{2}-2 x y \cos \phi_{1}\right)^{1 / 2}\right) \\
& \times \sin ^{n-2} \phi_{1} d \phi_{1} y^{n-1} d y,
\end{aligned}
$$


since

$$
\prod_{j=2}^{n-2} \int_{0}^{\pi} \sin ^{n-j-1} \phi_{j} d \phi_{j}=2 \pi^{(n-1) / 2} \Gamma\left(\frac{n-1}{2}\right)^{-1}
$$

Next, by the change of variables $t^{2}=x^{2}+y^{2}-2 x y \cos \phi_{1}$ we obtain

$$
\frac{t d t}{x y}=\sin \phi_{1} d \phi_{1}, \quad \frac{1}{2} x y \sin \phi_{1}=\Delta(x, y, t),
$$

so

$$
\begin{aligned}
& \int_{0}^{\pi} g\left(\left(x^{2}+y^{2}-2 x y \cos \phi_{1}\right)^{1 / 2}\right) \sin ^{n-2} \phi_{1} d \phi_{1} \\
& \quad=2^{n-3} \int_{|x-y|}^{x+y} g(t) \frac{\Delta(x, y, t)^{n-3}}{(x y)^{n-2}} t d t \\
& \quad=\Gamma\left(\frac{n}{2}\right)^{-1} \Gamma\left(\frac{n-1}{2}\right) \pi^{1 / 2} \int_{|x-y|}^{x+y} g(t) W_{x, y}^{(n-1)}(t) d t .
\end{aligned}
$$

Combining (7) and (8) gives (6).

It is clear that the correspondence $L_{r}^{1}\left(\mathbf{R}^{n}\right) \ni \tilde{f} \mapsto \Phi(\tilde{f})=f \in L^{1}\left(\mu_{n}\right)$ establishes the isometric isomorphism of the Banach algebras $L_{r}^{1}\left(\mathbf{R}^{n}\right)$ and $L^{1}\left(\mu_{n}\right)$ where the multiplication in the second one is defined by (6). The fact that $L^{1}\left(\mu_{n}\right)$, with $(6)$ as the multiplication, forms a commutative Banach algebra may be also verified without any difficulty using only (4) and the following identity:

$$
W_{x, y}^{(n-1)}(t) d t d \mu_{n}(y)=W_{x, t}^{(n-1)}(y) d y d \mu_{n}(t) .
$$

Moreover, (9) implies

$$
\int_{0}^{\infty} T^{y} g(x) h(x) d \mu_{n}(x)=\int_{0}^{\infty} g(x) T^{y} h(x) d \mu_{n}(x),
$$

for reasonable functions, e.g. $g \in L^{1}\left(\mu_{n}\right), h \in L^{\infty}\left(\mu_{n}\right)$.

Now, take $\bar{y} \in \mathbf{R}^{n}, \bar{y}=(1,0, \ldots$,$) . Since$

$$
\alpha(\tilde{f})=\int_{\mathbf{R}^{n}} \tilde{f}(\bar{x}) \exp (i\langle\bar{x}, \bar{y}\rangle) d \bar{x}
$$

defines a multiplicative functional on $L_{r}^{1}\left(\mathbf{R}^{n}\right)$, then also $\beta(f)=\alpha\left(\Phi^{-1}(f)\right)$ gives a multiplicative functional on $L^{1}\left(\mu_{n}\right)$. But, as one can easily verify

$$
\beta(f)=\int_{0}^{\infty} f(t) \phi^{(n-1)}(t) d \mu_{n}(t)
$$

so this and (10) gives

$$
\begin{aligned}
\int_{0}^{\infty} & \int_{0}^{\infty} f(x) g(y) \phi^{(n-1)}(x) \phi^{(n-1)}(y) d \mu_{n}(x) d \mu_{n}(y) \\
& =\int_{0}^{\infty} \int_{0}^{\infty} f(x) g(y) T^{y} \phi^{(n-1)}(x) d \mu_{n}(x) d \mu_{n}(y)
\end{aligned}
$$

for any $f, g \in L^{1}\left(\mu_{n}\right)$. By continuity of $\phi^{(n-1)}(t)$ we get

$$
\phi^{(n-1)}(x) \phi^{(n-1)}(y)=T^{y} \phi^{(n-1)}(x)
$$

for every $x, y \geq 0$, which proves $F(z)=G(z)$ for $z=n-1$. 
Since the assumptions of the Theorem are satisfied, to be precise, for the function $f(z)=\widetilde{F}(z+1)-\widetilde{G}(z+1)$, analytic for $\operatorname{Re} z>-1$, we conclude $\widetilde{F}=\widetilde{G}$ and also $F=G$ on $\operatorname{Re} z>0$.

\section{REFERENCES}

1. B. C. Carlson, Special functions of applied mathematics, Academic Press, New York, 1977.

2. D. L. Guy, Hankel multiplier transformations and weighted p-norms, Trans. Amer. Math. Soc. 95 (1960), 137-189.

3. E. C. Titchmarsh, The theory of functions, Oxford Univ. Press, London, 1939.

4. G. N. Watson, A treatise on the theory of Bessel functions, Cambridge Univ. Press, 1962.

Department of Mathematics, University of Georgia, Athens, Georgia 30602

Current address: Institute of Mathematics, University of Wroclaw, Pl. Grunwaldzki 2/4, 50-384 Wroclaw, Poland 